\title{
Design of a low-cost loading/unloading mechanism for processing stations in an automated production environment
}

\author{
Georg Egger ${ }^{1, *}$, Michael Riedl ${ }^{1}$, Erwin Rauch $^{2}$, and Dominik T. Matt ${ }^{1,2}$ \\ ${ }^{1}$ Fraunhofer Italia, Automation and Mechatronics Group, 39100 Bolzano, Italy \\ ${ }^{2}$ Free University of Bozen-Bolzano, Italy
}

\begin{abstract}
A loading/unloading mechanism was designed using the axiomatic design method. The mechanism is a standardized part of a smart factory currently implemented at Fraunhofer Italia research institute on behalf of the DeConPro research project, and as such is specified to fit any processing station. The mechanism is used in conjunction with an automated transport system, which carries standardized transport boxes on rails. The transport systems stop at the processing stations and shall be grabbed by the mechanism subject of this publication and dragged into the processing station, where. Also, the transport boxes must be fixed onto the shuttles of the transport system avoiding drops while moving, which is also part of the design exercise. Inside the processing station, the transport boxes shall be further movable in both directions perpendicular to the direction of the mechanism movement. The mechanism shall build compact especially in depth, and shall also be optimised in cost, as it is a recurring item in all processing stations. The resulting FR/DP decomposition lead to an decoupled design matrix up to second level, which allowed for choosing the right engineering sequence of the functions. The axiomatic design procedure helped considerably in finding the best concept for holding and handling the box. The further engineering steps benefit also considerably by the anticipated trade-off between alternatives for actuator types.
\end{abstract}

\section{Introduction}

Today, Industry 4.0 and cyber-physical production systems (CPPS) is an omnipresent buzzword in both traditional manufacturing and mass production and several research projects at Fraunhofer Italia are dedicated to this topic. Among other projects, Fraunhofer Italia is working on currently on a small-scale factory, which shall serve as a sandbox for applying decentralized control schemes to control the dynamic nature of production. Physically, the research infrastructure will consist of:

- an autonomous transport system as the common means for accessing all modules

- a laser cutting machine as a example for an digital production module

- an assembly station for a demonstration of the capabilities of a modern cooperative robotic arm

- a quality check module for automated routine checks

- a delivery station

The parts and the material is moved in so-called intelligent work-piece carriers (IWC), which are standardized containers and all handling operations will be designed around them. Hence, common to all modules is their interface to the transport system. As this is a recurring element, the impact on the overall system cost is of interest and was

\footnotetext{
*e-mail: georg.egger@fraunhofer.it
}

selected as worthy to be prototyped. Se Figure 1 for a conceptual illustration of the problem.

There are several ways of designing such a mechanism and it is easy to get lost among the number of requirements. In the past, the authors have made several successful experiences in using the Axiomatic Design method to structure and solve design problems in the field of assembly system design [1] [2] [3].

For this purpose, the axiomatic design method was selected as a systematic approach finding an answer to the principal design questions. See [4] and [5] for the definition of axiomatic design.

The remaining content of the paper is organised in the following chapters:

Chapter 2: Customer attributes The boundary conditions and expectations to the product are listed. In the absence of an external customer, the CAs are identified through a self-interview.

Chapter 3: Identification of Cs and n-FRs Among the CSs, contraints and non-functional requirements are identified. In particular the maximum accelerations are treated in detail.

Chapter 4: FR/DP decomposition This chapter holds the definition of FR0/DP0 and the first and second level decomposition. The decomposition adheres to the first axiom, the selection of appropriate physical implementations follows axiom 2 . 


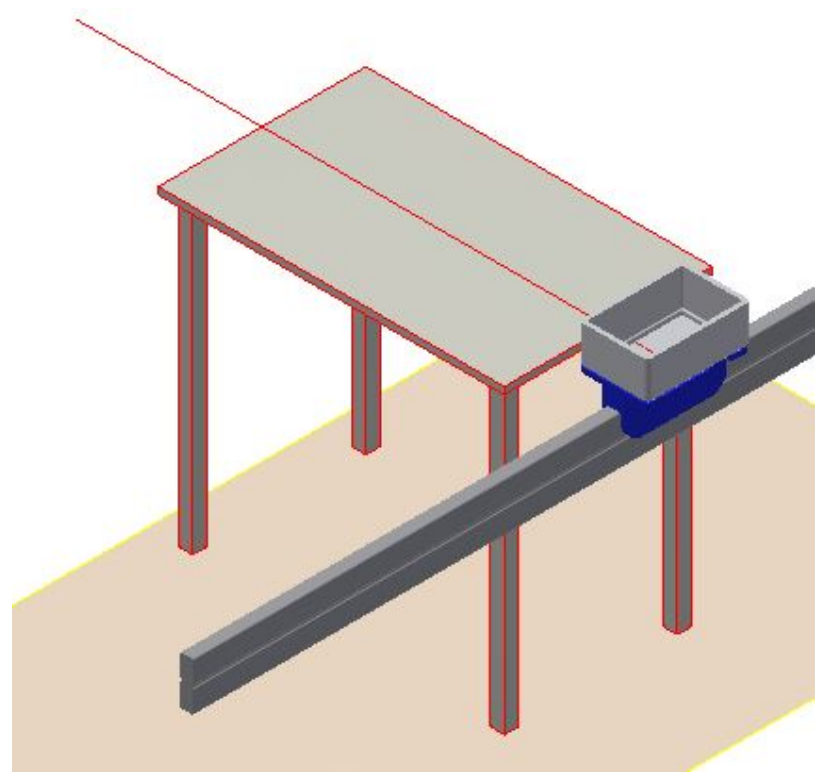

Figure 1. The design task comprises the supporting structure on the shuttles (blue) and the mechanism, which shall be integrated with the table-like object. The direction of movement is along the axis shown on the table.

Chapter 5: Discussion The approach to the problem is recapped, the benefits and limitations of the work is addressed.

Chapter 6: Outlook Planned follow-up activities on this topic.

\section{Customer attributes}

In the current application, there is no customer, as the mechanism is designed for the purpose of a research project. Hence the CAs are interpreted as user needs.

CA1 the transport boxes are $300 \mathrm{~mm}$ x $200 \mathrm{~mm}$ box Auer EG 32/12 HG (see Figure 2) filled no higher than $300 \mathrm{~mm}$. The boxes are to be positioned in the longitudinal direction on the shuttles, that is when the long side is parallel to the trajectory of the shuttles;

CA2 the boxes are brought on rail-based transport shuttles, which will stop at the given position quite precisely (1 mm or better);

CA3 the supporting structure holding the boxes on the shuttles shall be designed according to the needs of the mechanism;

CA4 the boxes shall not fall off the shuttles while being moved.

CA5 unlike on the shuttles, there is electrical power and compressed air available on the stationary side;

CA6 the mechanism shall be able to grab boxes, drag them up to $500 \mathrm{~mm}$ away from the shuttle (orthogonal to the rails) and to release them;
CA7 once retracted, the boxes shall be free to move in orthogonal to the mechanism movement (parallel to the rails);

CA8 compact build, particularly in depth;

CA9 the boxes are limited to $3 \mathrm{~kg}$ in mass, which can be assumed to be equally distributed throughout the volume envelope;

CA10 the shuttles move no faster than $55 \mathrm{~m} / \mathrm{min}$;

CA11 the shuttles accelerate from $0-30 \mathrm{~m} / \mathrm{min}$ in $0.5 \mathrm{~s}$ or slower, depending on the load;

CA12 the curves on the track shall have a radius of at least $220 \mathrm{~mm}$; and

CA13 there are more shuttles than stations.

\section{Identification of constraints and non-functional requirements}

The following constraints have been identified from the customer attributes:

C1 as container, Auer EG 32/12 HG [6] shall be used. The box with content weights $\max 3 \mathrm{~kg}$ and the mass can be assumed equally distributed;

C2 the longitudinal orientation of the boxes shall correspond to the movement of the shuttles on the rails;

C3 the shuttles will stop with a position accuracy of $1 \mathrm{~mm}$;

C4 compressed air and electrical power is available on the stationary side, no active power source available on the shuttles;

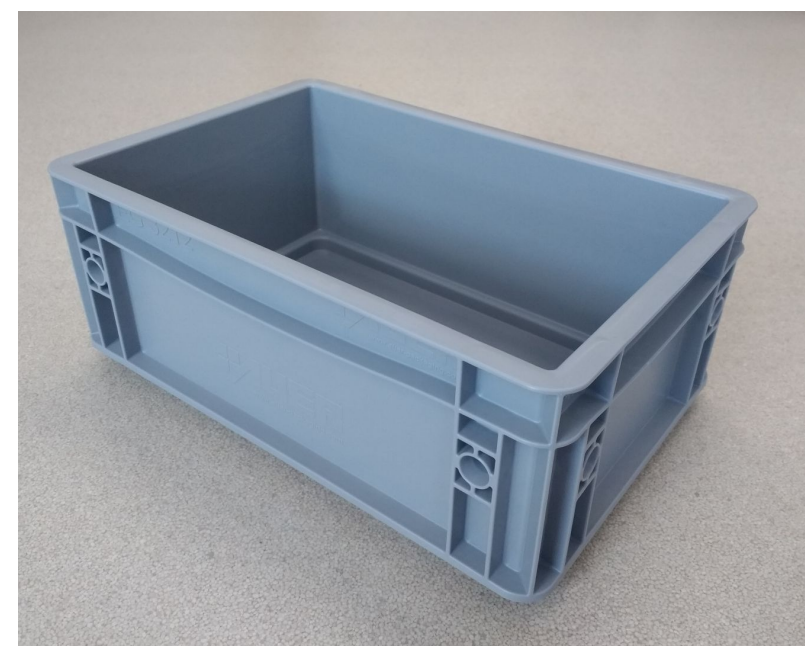

Figure 2. The stackable Euro container EG 32/12 HG from Auer Packaging $\mathrm{GmbH}$, which shall be used by the mechanism. The container features two handling holes on each lateral face, which may be used for grasping the box. 


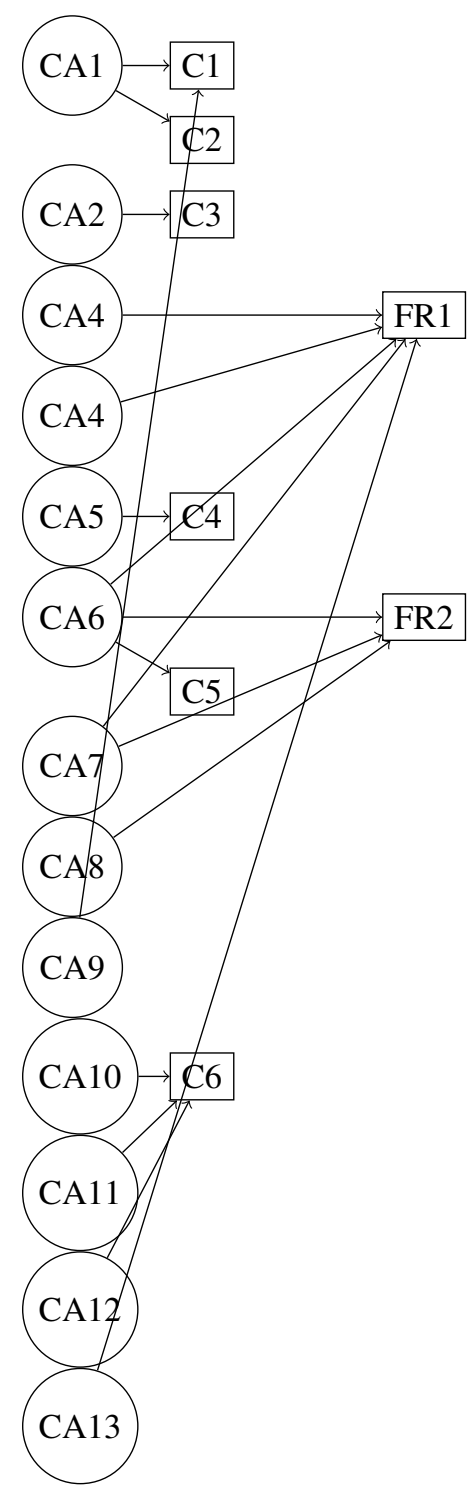

Figure 3. Assignment of customer attributes (CA) to constraints (C) and functional requirements (FR).

C5 the translation shall be $500 \mathrm{~mm}$ orthogonal to the rails on the same plane; and

C6 the maximum speed of the shuttles is is $55 \mathrm{~m} / \mathrm{min}$.

\subsection{Determining the accelerations acting on the box}

The maximum acceleration, that acts on the boxes while on the shuttle, can be derived from CA10, CA11, and CA12.

Two cases are to be distinguished:

- Longitudinal direction (starting and stopping)

- Transversal direction (moving through curves)

In the case of longitudinal acceleration, the shuttle accelerates from zero to $30 \mathrm{~m}$ per minute (CA10) in $0.5 \mathrm{sec}$ - onds (CA11). The acceleration is assumed to be constant:

$$
a_{\text {linear }}=\frac{\Delta v}{\Delta t}=\frac{30 \mathrm{~m} \mathrm{~min}^{-1}}{0.5 \mathrm{~s}}=\frac{30 \mathrm{~m}}{60 \mathrm{~s} \cdot 0.5 \mathrm{~s}}=1 \mathrm{~m} \mathrm{~s}^{-2}
$$

The longitudinal direction of the box corresponds to the longer side of the box $W$ (from CA1).

For the transversal acceleration, the shuttle travels with maximum speed through a curved element. Centrifugal forces act on the box and its content. Assuming a circularshaped curve with radius $r_{\min }$, the worst case acceleration is given by:

$$
a_{\text {circular }}=\frac{v_{\text {max }}^{2}}{r_{\text {min }}}=\frac{\left(\frac{55 \mathrm{~m} \mathrm{~min}^{-1}}{60 \mathrm{sin}^{-1}}\right)^{2}}{0.22 \mathrm{~m}} \sim 3.8 \mathrm{~m} \mathrm{~s}^{-2}
$$

\section{FR/DP decomposition}

The top level functional requirements and design parameters are defined as follows:

FR0 box handling for rail-based transport system

DP0 linear actuator with forklift mechanism

In the first level of the decomposition, two FR/DP pairs are identified:

FR1 hold and release of the transport boxes

DP1 frame and forklift

FR2 move the box perpendicular to the rails

DP2 linear actuator

The further decomposition is given in the following sub-chapters.

\subsection{FR1/DP1: Hold and release of the transport boxes}

There are numerous ways of holding the box on the shuttle, as requested by CA4. There are active ways, requiring the presence of some sort of energy source (primarily electrical or mechanical) on the shuttle, and passive ones, where the power required to overcome the locking mechanism is provided by the stationary side. Both ways, there is a strong coupling to the release mechanism on the stationary side.

The FR/DP pair can be decomposed further:

FR1.1 fix the boxes firmly on the transport shuttle

DP1.1 frame

FR1.2 release of the boxes

\section{DP1.2 forklift}

The partial design matrix shows a decoupled design, where the function of the box release (FR1.2) depends on the fixation device (DP1.1) and the design of the release mechanism (DP1.2).

$$
\left\{\begin{array}{l}
F R_{1.1} \\
F R_{1.2}
\end{array}\right\}=\left[\begin{array}{ll}
X & 0 \\
X & X
\end{array}\right]\left\{\begin{array}{l}
D P_{1.1} \\
D P_{1.2}
\end{array}\right\}
$$




\subsubsection{FR/DP 1.1: Fix the boxes firmly on the transport shuttle}

CA5 restricts the types of holding and release mechanisms to passive ones, which limits the possibilities significantly. In order to minimise the coupling factor between fixation and movement as much as possible, a simple interface is sought and found in a simple frame, embracing the base of the box. The introduction of this frame makes it impossible for the box to slip off the shuttle, the box however might tilt when sufficient acceleration is applied. The contained weight and speed of the boxes allow this frame to have a rather limited height. The maximum allowed height can be found by observing Figure 4, where $a$ represents in one case the constant acceleration and in the other the centrifugal acceleration. Both forces $F_{A}$ and $F_{G}$ can be composed in components acting along the line - defined by the center of rotation on the border of the frame and the center of gravity of the box - and perpendicular to it (direction of rotation). In the latter direction, both forces act in a contrary sense and the equilibrium point can be seen as the critical point corresponding to the tolerable acceleration.

The decomposition of $F_{A}$ and $F_{G}$ in the direction of rotation (see Figure 4) yields:

$$
a \sin \theta=g \cos \theta
$$

where $g$ is the gravitational constant and $a$ is the acceleration. For the angle $\theta$ one can find:

$$
\tan \theta=\frac{\frac{H}{2}-h}{\frac{W}{2}}=\frac{H-2 h}{W}
$$

Given a certain maximum acceleration $a_{\max }$ the step height $h$ must be at least:

$$
h \geq \frac{1}{2}\left(H-\frac{g W}{a_{\max }}\right)
$$

It can be seen, that the minimum required $h$ might as well be negative. In this case, the acceleration is not sufficient to lift the weight of the box at all.

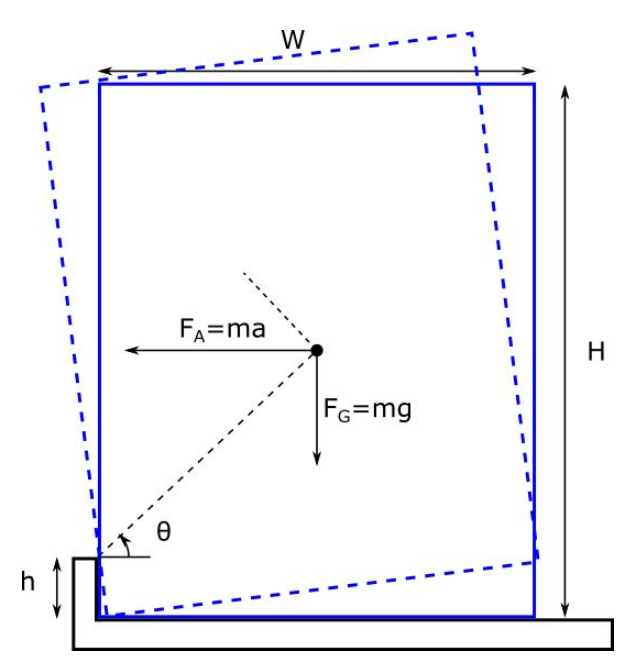

Figure 4. Acceleration of the box around the border frame.
C6 gives the numerical values for the worst case accelerations. Table 1 shows the results of the minimum height calculations. From the numerical values can be readily seen that the worst case is the circular motion.

Even in the worst case, the required minimum height for tilting is negative, which means that any height of the embracing frame will prevent falling off.

\subsubsection{FR/DP 1.2: Release of the boxes}

The box is supposed to be dragged over the table, perpendicular to the orientation of the rails. Before the box can be dragged, it must be raised above the edge of the supporting frame (FR/DP 1.1), in order to be released from the fixation.

Fundamentally, there are two approaches to this: the rise from the frame is performed by the shuttle or by the stationary side. The complexity seems manageable for both parties, but as CA5 states, there is no power available on the shuttles. Hence it is simpler allocating this function to the stationary side, also because there are more shuttles than stations in the system (CA13).

Another CA to be considered is CA7: Once retracted, the boxes must be able to move in the direction parallel to the rails (orthogonal to the linear actuator, see also Figure 6).

The process of releasing the box is hence twofold: First, release the box from its fixation on the shuttle and second, once retracted, release it from the mechanism and allow for movement parallel to the rails. As illustrated in Figure 7, there are two ways of performing this operation, the characteristics are discussed in Table 2.

Among the two possibilities the authors preferred option b), whose drawbacks could be overcome by design.

Table 1. Results minimum height calculation

\begin{tabular}{cccc} 
Scenario & $a_{\max }$ & $W$ & $h_{\min }$ \\
\hline Linear & $1 \mathrm{~m} \mathrm{~s}^{-2}$ & $300 \mathrm{~mm}$ & $-1.35 \mathrm{~m}$ \\
Circular & $3.8 \mathrm{~m} \mathrm{~s}^{-2}$ & $200 \mathrm{~mm}$ & $-0.1 \mathrm{~m}$
\end{tabular}

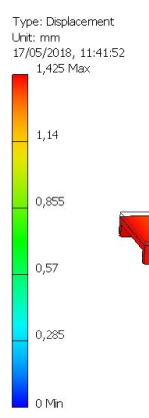

Figure 5. For estimating the order of magnitude of the deflection of a potentially feasible fork design, the displacement of a Ushaped aluminium profile has been carried out. The width of the sheet metal is $2 \mathrm{~mm}$, its length width $30 \mathrm{~mm}$ and the height $6 \mathrm{~mm}$. The worst case deflection amounts to less than $2 \mathrm{~mm}$ over a length of $200 \mathrm{~mm}$, which is sufficient to cover the width of the transport box [6]. 


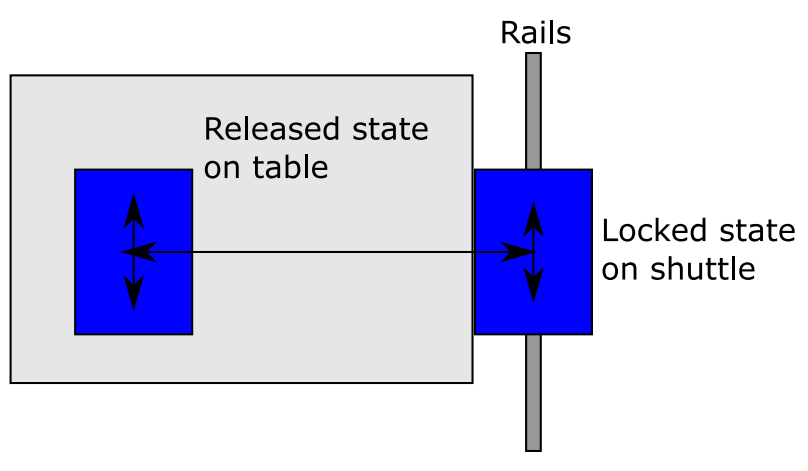

Figure 6. Longitudinal and orthogonal directions of movement. a)

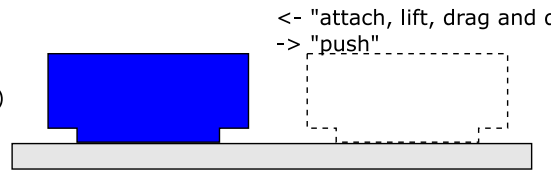

$<->$ "lift, move and let down"

b)

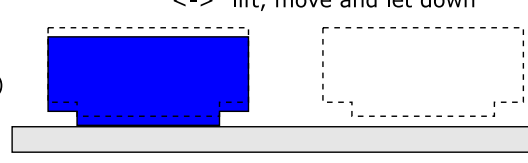

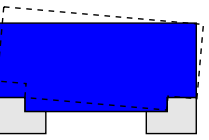

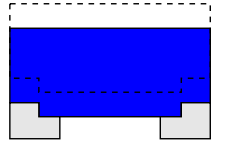

Figure 7. Design options for fetching the box. Option a) implies a hook mechanism, most conveniently engaging with the centering holes on the on the lateral sides of the box, in order to get hold to the box and to lift it. Option b) implies a forklift-like mechanism and grooves in the table for lowering the forks below the table surface.

Figure 8 shows the resulting design concept. A displacement analysis has been carried out, in order to determine an adequate material strength of the fork, see Figure 5 for result. The bending of less than $2 \mathrm{~mm}$ over the whole length shall be covered in the tolerances of the travel distance of the lifting device.

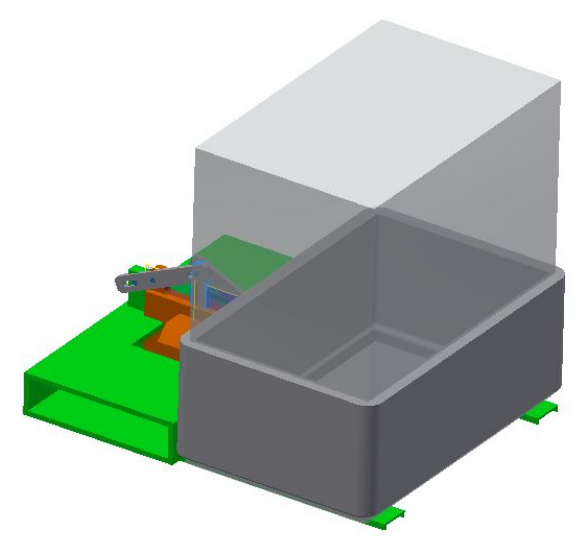

Figure 8. Lifting device carrying the box with volume envelope of its content.
Table 2. Comparing box fetching options

Option
phase, but lifting and gripping mechanism must be dimensioned to support half of the weight of the box

- friction between box and table must be overcome by a linear actuator and might result in a consumption of the boxes plastic material

- operation is asymmetric

b)

- requires forklift on the moving part of the mechanism

- is more robust against stop position of the shuttle

- requires grooves in the table for lowering the fork into

- the mechanism must carry the full weight of the box

- limited friction and hence no material consumption

- operation is symmetric

The rationale was guided by choosing the more simple option (axiom 2). The following thoughts were considered:

- the clamping of the box laterally required precise knowledge of the centering holes on the box. Inaccurate stopping position of the shuttles and/or potential obstructions represent unnecessary additional risk;

- even with option b), there is a way to transfer the weight of the box to the table surface through wheels; and

- compensating friction with motor power is inefficient.

\subsection{FR/DP 2: Linear actuator}

For the translatory movement on the stationary side, plenty of options exist, as both electric and pneumatic energy is available. The choice of the actuator type is not the only design parameter, there is also the choice of the placement of the actuator, which has great impact on complexity and performance.

The FR/DP pair can be decomposed furthermore:

FR2.1 actuator type

DP2.1 belt-type 
FR2.2 placement

DP2.2 below the table

The partial design matrix shows an uncoupled design.

$$
\left\{\begin{array}{l}
F R_{2.1} \\
F R_{2.2}
\end{array}\right\}=\left[\begin{array}{ll}
X & 0 \\
0 & X
\end{array}\right]\left\{\begin{array}{l}
D P_{2.1} \\
D P_{2.2}
\end{array}\right\}
$$

\subsubsection{FR/DP 2.1: Actuator type}

Pneumatic cylinders can be found often in industrial automation. Among their benefits is their simplicity in construction hence reduced cost, sturdiness in operation, achievable speed, self-guided, suitability for explosive atmospheres and hygienic-sensitive applications. Among the drawbacks there is the difficulty in controlling the position and the speed of the piston in between the final positions, limited force (hydraulic cylinders as an alternative), need for compressed air, noise. The actual force and piston speed depend on the pressure and flow rate of the compressed air. Figure 9 shows a pneumatic cylinder of suitable length. For implementation, electrically controlled valves, a pressure regulator, and magnetic limit switches are required, which neutralizes the cost-benefit of the actuator, if not shared with other cylinders.

Lead screw actuators (electric cylinders) are used similarly to pneumatic cylinders. Their popularity for certain is due to the relatively simple interfaces: 2 wires are sufficient for controlling the motor, reversing the polarity reverses the direction of movement. Internal limit switches bring the motor to a stop when it reaches the final positions. By measuring the current, the load on the motor can be easily monitored and overload can be avoided by electrical fuses. Due to the lead screw principle, the actuator is self-locking. Intrinsic to the operating principle is however also the slow operation of the piston. Similar to a pneumatic cylinder the device requires significant volume

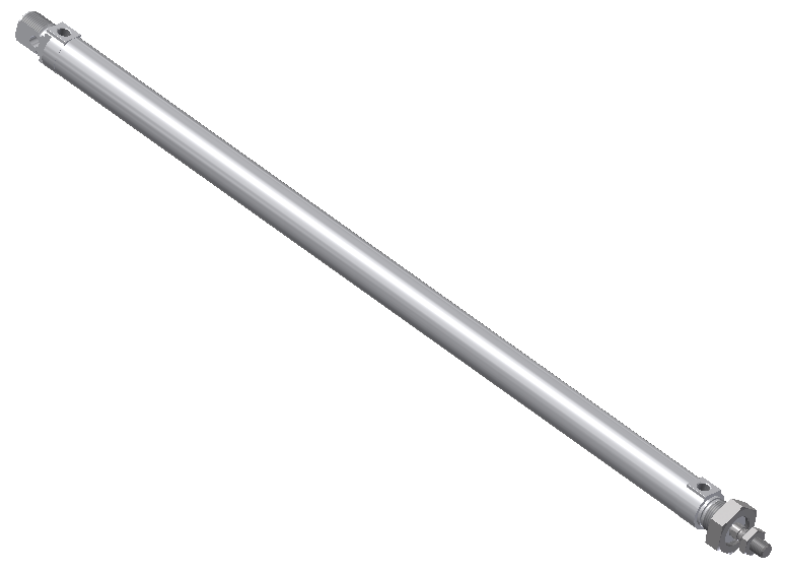

Figure 9. Festo DSNU [7] pneumatic cylinder in $500 \mathrm{~mm}$ length with $25 \mathrm{~mm}$ piston diameter. The cylinder is shown in the retracted state. When the piston is extended, the overall length almost doubles. when fully extracted, therefore the available stroke length is rather confined.

The Linear-Mech UBA-0 [8] is an example of a lead screw actuator with motor assembly for loads up to $420 \mathrm{~N}$ and linear speeds of up to $500 \mathrm{~mm} / \mathrm{s}$. The device can be equipped with mechanical and/or magnetic limit switches and with an encoder for position feedback.

Direct drive linear motor technology has been around for decades, it's implementation has however been limited due to the associated complexity. Linear motors are electrical motors where the windings are laid out on a linear axis. The windings are powered and driven by power electronics, which can be controlled to a good precision just from the stator side. The dynamics of such a system can be impressive, which comes at the price of complexity and power consumption. Linear motors can be found in applications with extraordinary dynamic requirements, particularly in spatially limited environments.

The LDC-series of Allen-Bradley [9] is a commercial example of such a device. It delivers up to $10 \mathrm{~m} / \mathrm{s}$. A single rail is available with lengths of up to $600 \mathrm{~mm}$, several rails can be connected to cover larger distances. The rails require individual high-voltage power supplies and the maximum actuation force depends strongly on the employed cooling technique.

Rack and pinion drives are used for more than 100 years to transform rotation in linear motion by the use of a sprocket (the pinion) and a geared track (the rack). The transmission ratio is determined by the gear ratio, which can be adapted to the needs of the application either directly or in a wider range through gears like worm or epicyclic gear boxes. A linear actuation mechanism must provide sufficiently strong guidance between the elements whilst allowing them to move in the desired direction easily, otherwise the pinion is likely to slip or the friction to be overcome is too high.

Wittenstein produces many rack and pinion drive components with planetary gearboxes [10]. The portfolio of their value line starts with loads of $100 \mathrm{~kg}$ and the maximum speeds are given to about $2-7 \mathrm{~m} / \mathrm{s}$. The application is mainly in industrial machinery.

Rigid chain actuators use interlocking chain elements in order to resist to both drag and compression. As a consequence, the rigid chain actuator behaves similarly to a rack and pinion actuator, while occupying less space in the retracted state. As a consequence rigid chain actuators can be found in cases where rack and pinion actuators would obstruct useful space. Rigid chain actuators are available in different sizes.

A chain actuator can be seen as a special type of a rack and pinion drive. A pinion drives an interlocking chain, which is resistant to both drag and compression in at least one direction and can often be found in window opening mechanisms. For example, Windowmaster WMU 836 [11] is a $300 \mathrm{~N}$ model and operates at up to $11 \mathrm{~mm} / \mathrm{s}$. The device is tested to 10000 movements over its lifetime. 
Belt driven actuators can be found in many spaceconfined applications, where precisely controlled movements are required. The use of timing belts (synchronous belts) ensures linearity in between final positions, and hence by simply controlling the revolutions of an electrical motor the position is defined. The motion is smooth and depending on the mobile mass also reasonably fast. Belt drives are used with medium travel distances, limited mainly by the elasticity of the belt on the upper side and the dimensions of the sprockets on the lower side.

A belt driven linear axis uses a timing (synchronous) belt and sprockets, interconnected by stiff rails, which guide a sled. The motor can be directly connected or through a gearbox. An example is the Igus drylin E series entry-level model [12], which features a directly connected NEMA23 stepper motor and is capable of up to $2 \mathrm{~m} / \mathrm{s}$ for a load of $100 \mathrm{~N}$.

With many options available, the best option can be selected by performing a trade-off in the sense of axiom 2 of the axiomatic design theory. The constraints of the particular application have been taken into consideration and included in the qualitative weighting of the individual options (see Table 3). Promising options are pneumatic and electrical cylinders, however as the spatial demands are stringent, the length of the cylinders in the extracted state represents an issue. Among the other options, the belt actuator fits best in the set of requirements and is selected as a baseline. Having considered the loads which are to be expected, a stepper motor was selected as the active unit. Other options would have considered servo motors or induction motors, which however need encoders, which would drive up the cost and complexity unnecessarily.

\subsubsection{FR/DP 2.2: Placement}

The placement of the linear actuator is relevant, as it might obstruct the movement of the box. There are 3 fundamental options:

1. lateral to the perpendicular movement of the box

2. above the volume envelope of the box (crane-like)

3. below the table surface

The first option, however, restricts the further movement of the box to the side of the mechanism and hence is not compliant to CA7. Also, it is not in the spirit of CA8,

Table 3. Summary of linear actuator options

\begin{tabular}{|c|c|c|c|c|c|c|}
\hline Option & 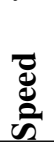 & ְָ & $\underset{⿱ 乛}{\Xi}$ & & $\overrightarrow{\tilde{\theta}}$ & 志 \\
\hline Pneumatic cylinder & + & 0 & - & + & 0 & + \\
\hline Lead screw & - & + & - & + & + & + \\
\hline Linear motor & + & - & + & - & - & - \\
\hline Rack and pinion & 0 & + & 0 & 0 & - & 0 \\
\hline Rigid chain & - & - & + & + & 0 & 0 \\
\hline Timing belt & + & 0 & + & 0 & 0 & ++ \\
\hline
\end{tabular}

as it occupies additional area in the working plane. Hence the lateral placement is rejected.

Two options remain, above the volume envelope, or below the table. It seems straightforward that the option below the table is less complex, as the overhead option would require structural elements in another plane over the boxes and their contents, which so far was not required. It is noteworthy, that this choice is independent of the release mechanism (DP1.2), even though other release principles could have a coupling.

We have seen that the placement of the linear actuator below the table seems reasonable. As a consequence, the motion must, however, be transferred to the top of the table. This requires a slot in the table along the direction of the linear axis, and a fin transfers the force to the top (Figure 10 and Figure 11).

\subsection{Design matrix}

The complete design matrix follows from the decompositions in the previous chapters:

$$
\left\{\begin{array}{c}
F R_{0} \\
F R_{1} \\
F R_{1.1} \\
F R_{1.2} \\
F R_{2} \\
F R_{2.1} \\
F R_{2.2}
\end{array}\right\}=\left[\begin{array}{ccccccc}
X & & & & & & \\
& X & & & & & \\
& & X & & & & \\
& & X & X & & & \\
& & & & X & & \\
& & & & & X & \\
& & & & & & X
\end{array}\right\}\left\{\begin{array}{c}
D P_{0} \\
D P_{1} \\
D P_{1.1} \\
D P_{1.2} \\
D P_{2} \\
D P_{2.1} \\
D P_{2.2}
\end{array}\right\}
$$

The overall design matrix is decoupled, with offdiagonal coupling only for FR1.2 between DP1.2 and

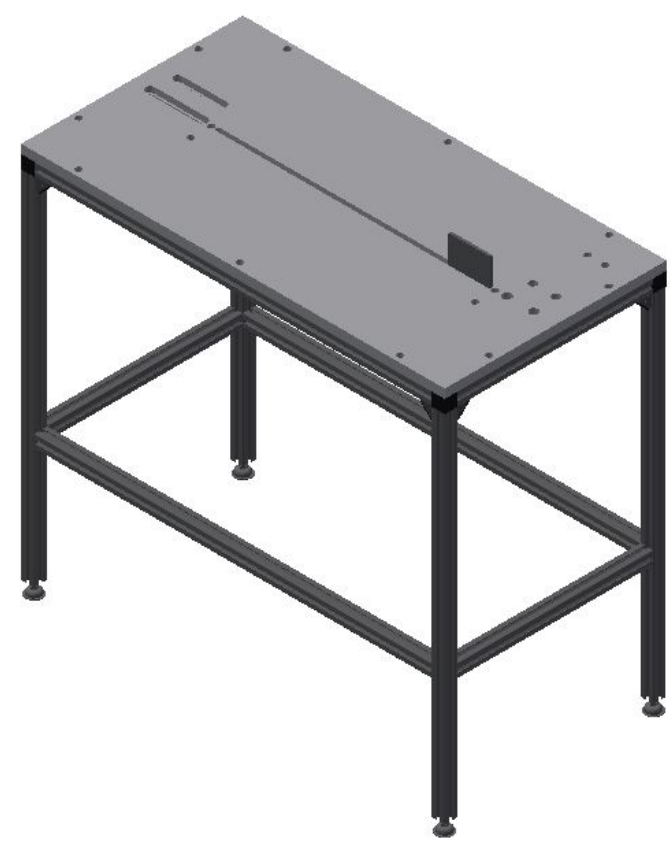

Figure 10. The final concept for the linear actuator integrated in the table. The fin transfers the motion to the top of the table, all active elements can be placed below the level where the boxes are handled. 


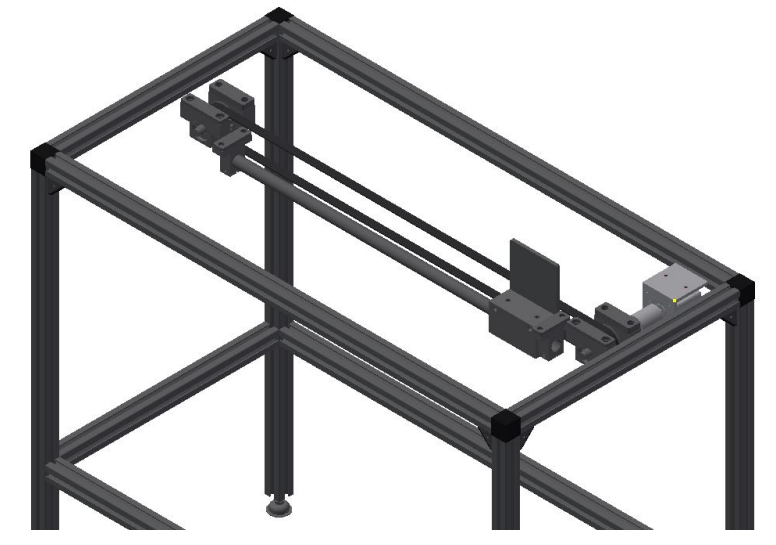

Figure 11. The table top has been removed and the rails, the stepper motor, the timing belt, the sprockets and various support brackets are revealed..

DP1.1. The resulting dependency can be managed by sequentially engineering the FR1.1 (fix box on shuttle) and then FR1.2 (release). Other than this, there are no couplings to be considered.

\section{Discussion}

At the begin of the design process, the authors thought of the problem as a single function: The idea was to perform the lifting and movement with a single actuator. The actuator would push and drag a fork-like construction, guided by lateral slots. Those slots would vary in height over the stoke length of the actuator, and control so the height and angle of the fork. At first glance, the initial idea appeared simple, because it could fulfil the requirements with just a single actuator. Looking at the approach more in detail, it turned out that the concept was more complex as expected if feasible at all: The lifting and putting down at the shuttle requires two different profiles of the slots, otherwise the fork would hit the box when trying to fetch the box from the shuttle. Different profiles require some sort of switch, which is de facto another function, so the appealing single function was proved infeasible. Furthermore the required steepness of the slots profile was also questionable, as the whole amount of lifting must occur in a narrow range of the actuator stroke, resulting in an oversized actuator with respect to the mass of the box.

The difficulties with the first design attempt lead the authors to the application of axiomatic design, which rapidly lead to the decomposition into two primary functions: Hold/release, and linear movement of the box. The result of the axiomatic design application is a decoupled design matrix, which means that the functions shall be developed in sequence in order to avoid unnecessary iterations, the hold/release function first, then the movement.

Both functions can be adjusted independently from each other. An example for a possible adaptation could be the change of the transport system to a faster one. The dynamics of it could require the fixation of the boxes also in the height dimension, so the forklift solution to release them would no longer be feasible. These aspects are covered by FR/DP 1.1 and 1.2 and as long as the other constraints remain the same, the FR/DP 2 remain entirely unaffected.

The physical solution as result of the axiomatic design process is however determined to a large degree by the boundary conditions of the application, particularly the transport box, transport system characteristics and volume envelope of the material. For example, the given application requires to release the boxes once they reach their final position on the table (CA7). This might not be the case elsewhere, which would allow for a simpler principle of grasping (i.e. dragging instead of lifting). Hence care must be taken when transferring the results to a different application.

\section{Outlook}

The work has shown that by applying axiomatic design methodology in a design phase of a coupled system has lead to a robust design concept. The design concept will now further engineered to more detailed technical specifi-

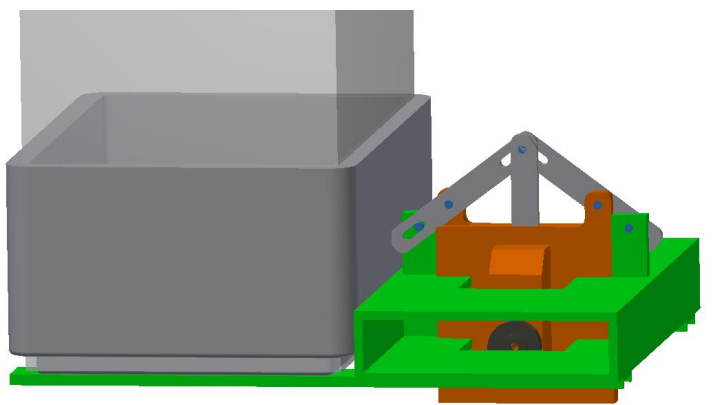

Figure 12. The final concept of the lifting actuator in the lowered state. Through a rod, the force required to lift the box is transferred. Via a symmetrical lever design, the force required to lift the box is applied to both sides of the fin, which should help to prevent wedging. Also visible, the wheels which run on the table top surface.

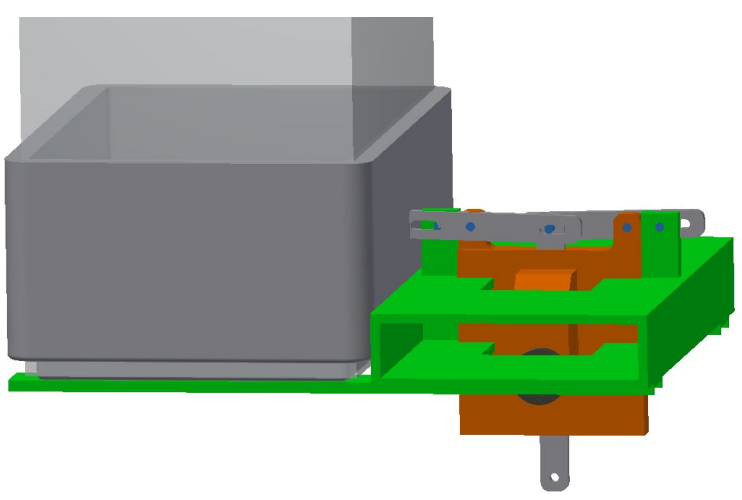

Figure 13. The lifting mechanism in the raised state. The rod connecting to the bottom of the plate connects to a cylinder, which is mounted below the table. This way, no active elements must be placed above the table surface. 
cations. The testing of the prototype is scheduled for the end of 2018.

This paper has been supported by funding from the European Regional Development Fund ERDF 20142020 under the grant agreement with project DeConPro FESR1017 (CUP B56J16001730001).

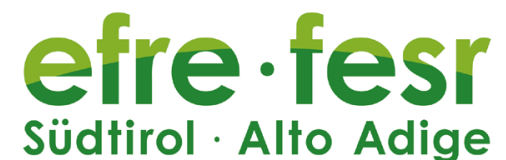

Europäischer Fonds für regionale Entwicklung Fondo europeo di sviluppo regionale

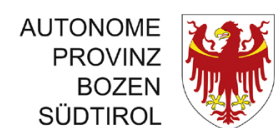

PROVINCIA AUTONOMA DI BOLZANO ALTO ADIGE

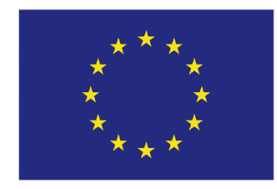

EUROPEAN UNION

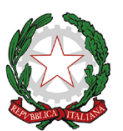

\section{References}

[1] D.T. Matt, Design of a scalable assembly system for product variety, in Assembly Automation (2013), Vol. 33, pp. 117-126, iSSN: 0144-5154, doi: 10.1108/01445151311306627

[2] D.T. Matt, Application of Axiomatic Design principles to control complexity dynamics in a mixedmodel assembly system: A case analysis, in International Journal of Production Research (2012), Vol. 50, pp. 1850-1861, iSSN: 0020-7543, doi: 10.1080/00207543.2011.565086

[3] D.T. Matt, Template based production system design, in Journal of Manufacturing Technology Management (2008), Vol. 19, pp. 783-797, iSSN: 1741038X, doi: $10.1108 / 17410380810898741$

[4] N.P. Suh, The principles of design (Oxford university press, 1990)
[5] N.P. Suh, Axiomatic design: Advances and Applications (Oxford university press, 2001)

[6] Auer Packaging GmbH, Euro container EG 32/12 HG product page (2018), https://www . auer-packaging.com/ Euro-containers-solid/EG-3212-HG.html

[7] Festo, Standard cylinders DSNU/DSNUP/DSN/ESNU/ESN, ISO 6432 catalogue, https://www. festo.com/cat/en-gb_ gb/data/doc_engb/PDF/EN/DSNU-ISO_EN.PDF (2015)

[8] Linear-Mech S.r.1., UBA 0 ball screw linear actuator, http://www.linearmech.it/linearmech/ azienda/azienda/documenti_prodotti/uba $\theta_{-}$ eng.pdf

[9] Rockwell Automation Inc., Allen-Bradley Kinetix Linear Motion Specifications MPSeries, LDAT-Series, LDC-Series, LDL-Series, http://literature. rockwellautomation. com/idc/groups/literature/documents/td/ knx-td002_-en-p.pdf (2018), KNX-TD002BEN-P

[10] Wittenstein, alpha Linear Systems Product Catalogue, https://www.wittenstein.de/ download/alpha-linear-systems-en.pdf (2018)

[11] WindowMaster A/S, WMU 836 chain actuator 300N product sheet (2018), https: //www . windowmaster. com/Admin/Public/ DWSDownload . aspx?File=\%2fFiles\%2fFiles\% 2fPDF\%2fdatablade\%2fWMU_836-n_data_ UK-13.pdf

[12] igus GmbH, drylin E electric linear axes and linear drives with motor (2018), toothed belt axis size 1040 